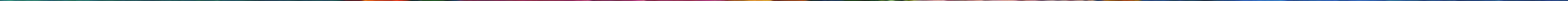




\section{El Envejecimiento en Honduras: una Caracterización Sociodemográfica del Adulto Mayor}

Marysabel Zelaya Ochoa*

RESUMEN. El envejecimiento es un fenómeno ligado a la llamada transición demográfica que se evidencia en la declinación de las tasas de mortalidad y fecundidad y sus efectos en la estructura de la población. Algunos elementos como el acceso a los servicios de salud, adelantos en la medicina, alto nivel educativo, saneamiento ambiental son factores que están relacionados con el aumento de la esperanza de vida, e incide a su vez en el envejecimiento gradual de la población. Las características sociodemográficas de la población adulta mayor en Honduras refleja que este grupo se encuentra en condición de vulnerabilidad. En el contexto de este estudio esta vulnerabilidad es evidente en los bajos niveles educativos, alta participación en el mercado laboral ligado en cierta medida a la escasa cobertura en seguridad social. Los datos reflejan un aumento de la longevidad entre la población hondureña, sin embargo, esto no sólo debe traducirse en vivir más años, sino también en tener calidad de vida.

Palabras clave: envejecimiento, población adulta mayor, esperanza de vida, educación, ingresos, rama de actividad, situación de empleo.

ABSTRACT. The aging is a phenomenon tied to the called demographic transition that showed evidence in the decline rates of mortality, fecundity and its effects in the population structure. Some elements like the access of health services, advances in medicine, high educative level, and environmental sanitation are facts that have a relationship with the increase of life's hope, and index turn the gradual aging of population.

The socio demographic characters of older population in Honduras reflect that this group is in the conditions of vulnerability. In this study context the vulnerability is really clear in the low educative levels, high participation in the labor market tied in some way to the low social security coverage. Dates showed an increase of longevity in Hondurans population, however, this has not to be translating in living more years, but also in have a life with quality.

Keywords: Aging, older population, life's hope, education, incomes, industry, employee situation.

\section{Introducción}

El envejecimiento de la población ha tomando notoriedad en los últimos años y es un fenómeno ligado a la llamada transición demográfica que se evidencia en la declinación de las tasas de mortalidad y fecundidad y sus efectos en la estructura de la población.

Algunos elementos como el acceso a los servicios de salud, adelantos en la medicina, alto nivel educativo, saneamiento ambiental son factores que están relacionados con el aumento de la esperanza de vida, que incide a su vez en el envejecimiento gradual de la población.
Datos del Instituto Nacional de Estadística (INE) entre los años 2001, 2005 y 2010 muestran que en Honduras se presenta un crecimiento de la población adulta mayor reflejando una tendencia al incremento de la población de 60 años y más.

En el contexto de país, la población adulta mayor se encuentra en una situación vulnerable puesto que sufren condiciones de pobreza, marginación, falta de seguridad social, disminuyendo notablemente su calidad de vida.

Esta investigación tiene como objetivo presentar una caracterización sociodemográfica de este grupo poblacional que permita observar su comportamiento en el

Recibido: septiembre 2012/ Aceptado y versión final: octubre 2012.

* Docente investigadora de la Maestría en Demografía y Desarrollo, Universidad Nacional Autónoma de Honduras 
periodo 2001, 2005 y 2010 en aspectos como: estructura de la población, características educativas y características económicas.

Se pretende que este estudio genere inquietudes y despierte el interés de los tomadores de decisiones, sobre todo porque este grupo poblacional aumentará su participación en relación a la población total lo que implica a su vez una demanda duplicada de servicios para la tercera edad que en el contexto del desarrollo humano no puede pasar desapercibida.

\section{Metodología}

Este estudio se desarrolló bajo un enfoque descriptivo, se hizo una revisión bibliográfica para desarrollar un contexto teórico del tema de envejecimiento a nivel mundial, latinoamericano y nacional.

Las variables consideradas fueron: estructura de la población seleccionando el total de la población según sexo, área de residencia, situación conyugal, jefatura de hogar e índice de envejecimiento. En las características educativas se eligió el analfabetismo y nivel educativo. Para las características económicas, condición de actividad económica, situación de empleo, rama de actividad e ingresos.

La fuente de datos utilizada en esta investigación son las Encuestas Permanentes de Hogares de Propósitos Múltiples 2001, 2005, 2010, la unidad de análisis es la población de 60 años y más.

\section{Objetivo General}

Presentar las características sociodemográficas de la población adulta mayor en Honduras en el periodo 2001, 2005 y 2010.

\section{Marco Teórico}

\subsection{El Concepto de Adulto Mayor}

Existen diversas definiciones del concepto de adulto mayor entre las que destacan aquellas de carácter cien- tífico, biológico, médico, geriátrico, y psicológicas, entre otras, cada una de ellas centra su definición en el contexto de su especialidad.

El programa de las Naciones Unidas define como adulto mayor a toda persona mayor de 65 años, en los países desarrollados, y el caso de los países en desarrollo, a toda persona mayor de 60 años.

La Organización Mundial de la Salud divide a los adultos mayores en tres subgrupos: de 60 a 74 años, se consideran personas de edad avanzada, de 75 a 90 años ancianas o viejas, y las que sobrepasan los 90 años, se les llama grandes viejos o grandes longevos.

En el contexto de esta investigación se consideran personas adultas mayores o personas de la tercera edad a todas aquellas que han alcanzado 60 años ó más.

\subsection{El Proceso de Envejecimiento}

El envejecimiento de la población es un fenómeno que experimenta gran parte de los países a nivel mundial, este se refleja en un cambio en la estructura por edades de la población, que genera a su vez un incremento en los índices de envejecimiento, razón por la cual el fenómeno comienza a inquietar a los gobiernos sobre todo por las implicaciones que genera a nivel económico, social, demanda de servicios de salud y en seguridad social entre otras.

La Organización Mundial de la Salud define el envejecimiento como el proceso fisiológico que comienza en la concepción y ocasiona cambios característicos para las especies durante todo el ciclo de vida.

El envejecimiento es un proceso que se desarrolla gradualmente entre la población. Las personas envejecen a medida que en su tránsito por las diversas etapas del ciclo de vida gana en años, una población envejece cada vez que las cohortes de edades mayores aumentan su ponderación dentro del conjunto (Villa, M y Rivadeneira, L 2000: 24-26).

El envejecimiento demográfico es el resultado del avance social y económico que incrementó la esperanza de vida y de una política de población que disminuyó la fecundidad. 


\subsubsection{El proceso de Envejecimiento en el Mundo}

La vida promedio de la población está aumentando y lo seguirá haciendo en las décadas por venir. Los datos muestran como los cambios en la mortalidad de la población a nivel mundial se reflejan en una mayor sobrevivencia, la cual aumentará considerablemente en la primera mitad de este siglo, al pasar de alrededor de 65 años en 2000-2005 a 74 años en 2045-2050. Actualmente, la esperanza de vida de las mujeres (67.6 años) es superior en alrededor de cinco años a las que registran los varones (63.3 años). Cabe resaltar que en los países más desarrollados, la esperanza de vida al nacimiento, en promedio fue de casi 76 años en el quinquenio 2000-2005 la cual podría aumentar a 81 años a mediados de siglo XXI, a la vez que en los países en desarrollo se estima que aumentará de 63.4 a 73.1, durante el mismo periodo (CONAPO, 2000:12).

Actualmente, en los países desarrollados y en aquellos con economías en transición vive el porcentaje más alto de adultos mayores, para el caso en países como Islandia, Noruega, Suiza, Canadá, Estados Unidos, Japón y Nueva Zelanda sólo aumenta el número de personas mayores, mientras disminuye el de niños/as y población en edad de trabajar.

Entre 1950 y 1980 el porcentaje de adultos mayores con respecto a la población total se mantuvo alrededor de $8 \%$, mientras que en 2000 ascendió a 10\%. Bajo las tendencias previstas del envejecimiento demográfico mundial, se estima que en 2030 este porcentaje ascenderá a 16.6\% y en 2050 a 21.4\% (Gráfico No.1). En otras palabras, mientras que en año 2000 una de cada diez personas en el mundo era un adulto mayor, a mitad de siglo será una de cada cinco (CONAPO, 2000:13).

\subsubsection{Envejecimiento en América Latina}

En el caso de América Latina y el Caribe el número de personas de 60 años o más aumentará sostenidamente en las próximas décadas. La brusca caída en las tasas de fecundidad y el mejoramiento en la salud de los adultos mayores que se observa en América Latina durante los últimos 50 años, ha tenido como resultado el hecho que
Gráfico No. 1. Porcentaje de la Población Mundial de 60 Años y Más, Respecto a la Población Total 1950-2050

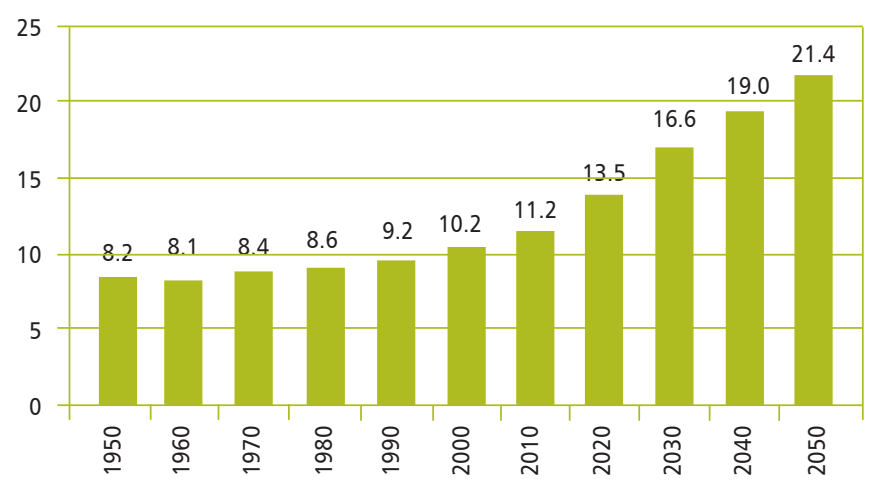

Fuente: World Population Prospects: The 2002 revision.

las personas mayores de 60 años representen hoy en día un crecimiento notable. Si bien las personas de 60 años o más representaban el $4.4 \%$ de la población total en el 1980, para el 2010 esta cifra aumentó a 6.9\% y se proyecta que para el 2030 este grupo alcance 12.2\% (Gráfico No.2).

La población de América Latina es considerablemente más joven que la de Estados Unidos, Japón y la mayoría de los países europeos. En Estados Unidos, en el 2000, las personas de 65 ó más años representaban al 13\% de la población, mientras que el $17 \%$ de la población de Japón está compuesta por personas mayores. Se espera que estos porcentajes aumenten en ambos países durante los próximos años (Population Reference Bureau, 2003).

Gráfico No. 2. América Latina: Población de 60 años y Más (En porcentaje)

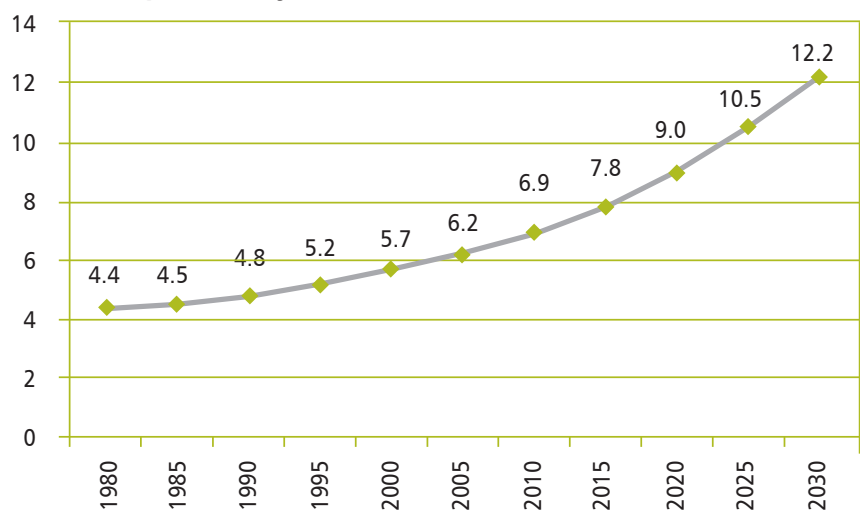

Fuente: Elaborado con datos de la CEPAL, Observatorio Demográfico No. 7 Abril 2009. 
El envejecimiento de la población en América Latina y el Caribe es el resultado de los éxitos obtenidos en materia de salud pública, que redujeron la mortalidad infantil durante el decenio de los años cuarenta, cuando los índices de fecundidad eran altos. Lo anterior estuvo seguido por las disminuciones bruscas en la fecundidad durante los años setenta y los incrementos de la esperanza de vida a la edad de 60 años durante la década de los noventa.

\section{Características Sociodemográficas de la Po- blación Adulta Mayor en Honduras}

\subsection{Estructura de la Población Adulta Mayor Según} Sexo

Al estudiar la estructura de la población en Honduras según edad, refleja un país con una estructura poblacional muy joven donde los niños, niñas y jóvenes representan para el 2012 un 48\% de la población ${ }^{1}$, esta característica es propia de los países en desarrollo. Sin embargo, la estructura por edad muestra ligeros cambios de la población que conducen a estudiar temas como el envejecimiento demográfico.

Durante el periodo de estudio seleccionado se observa que este grupo poblacional tuvo un crecimiento lento pero sostenido, para el caso en el 2001 representaba un $6.7 \%$ de población, en el 2005 era de $7.3 \%$ y para el 2010 fue de $8.8 \%$, presentando un crecimiento de 2.1 puntos porcentuales (Gráfico No.3).

Aunque Honduras ha entrado en un proceso de transición demográfica tardío, los pequeños cambios en la estructura de la población por edad también inciden en el grupo poblacional mayor de 60 años.

Por condición de género, la población adulta mayor en el país está constituida en su mayoría por mujeres, esto en parte se explica porque la mujer tiene una esperanza de vida más alta respecto a los hombres, además los avances médicos y sanitarios han logrado reducir la
Gráfico No. 3. Honduras: Población Menor y Mayor de 60 Años (En porcentaje)

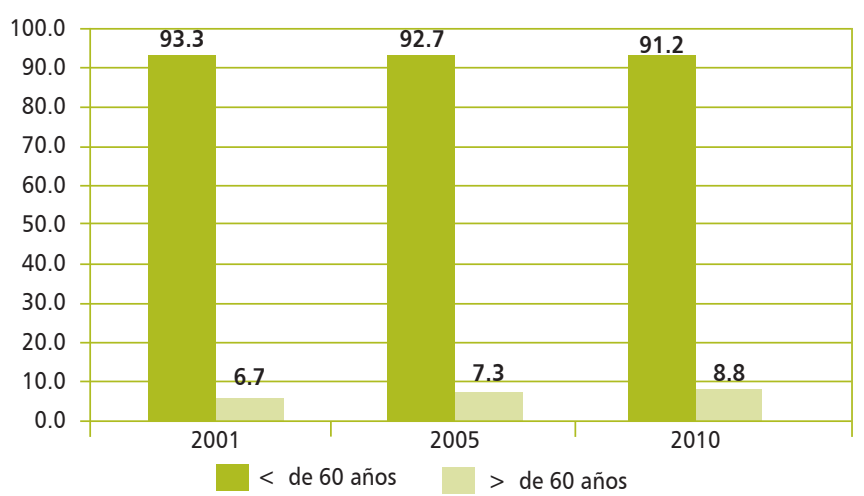

Fuente: Elaborado con datos del INE, EPHPM, 2001. 2005, 2010

mortalidad y disfrutar de mayores índices de esperanza de vida (Gráfico No.4).

Según proyecciones realizadas por $\mathrm{CEPAL}^{2}$ la esperanza de vida en Honduras, mostrará incremento pasando de 73 años en el quinquenio 2010 - 2015 a 76 años para el quinquenio 2030-2035. Se observa a la vez que es la mujer la que presenta mayor esperanza de vida respecto al hombre (Gráfico No.5).

Al desagregarlos según área de residencia la mayor parte de la población adulta mayor se ubica en el área rural. En promedio en los tres años objeto de estudio, $53.7 \%$ de la población mayor de 60 años vivía en el área

Gráfico No. 4. Honduras: Población Adulta Mayor Según Sexo (En porcentaje)

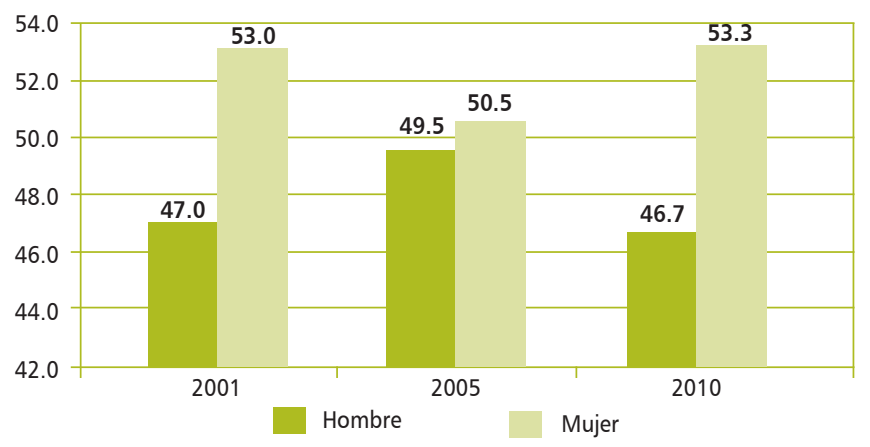

Fuente: Elaborado con datos del INE, EPHPM, 2001. 2005, 2010.

1. Cálculos realizados según proyecciones de población del INE.

2. CEPAL, Aérica Latina y el Caribe, Observatorio Demográfico, año VI, No12, Octubre 2011. 
Gráfico No. 5. Honduras: Población Adulta Mayor Según Esperanza de Vida y Sexo

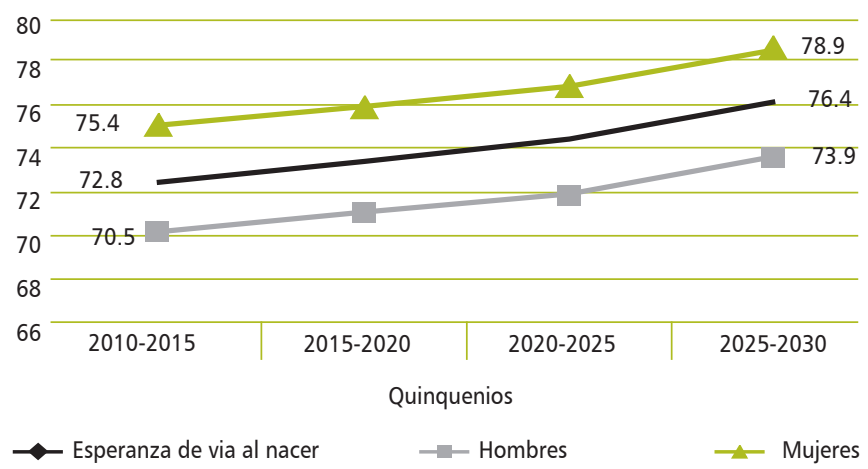

Fuente: Elaborado con datos CEPAL, Octubre 2011.

rural, muy similar a la tendencia nacional que para el mismo periodo fue de $53.9 \%$. Los porcentajes en los tres años estudiados 2001, 2005 y 2010 son muy similares y no reflejan diferencias notables entre el área urbana y rural (Gráfico No.6).

Gráfico No. 6. Honduras: Población Adulta Mayor, Según

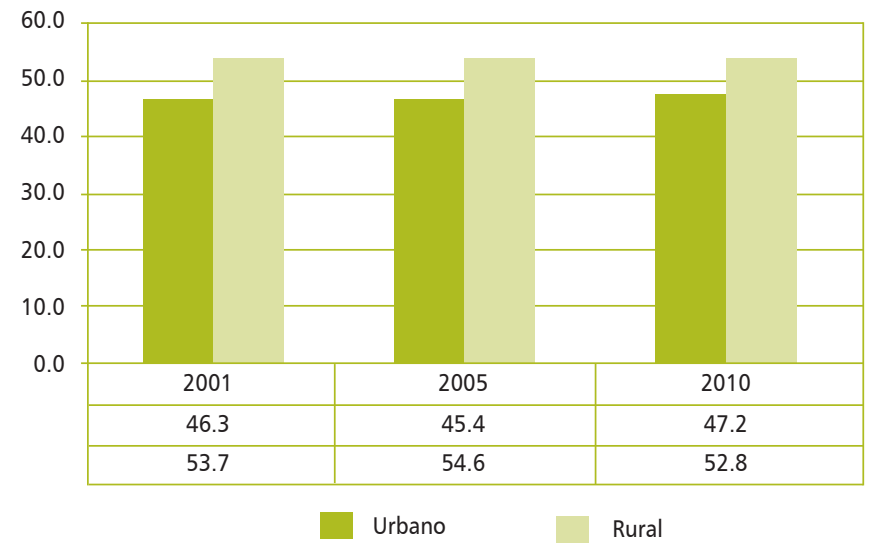

Fuente: Elaborado con datos del INE, EPHPM, 2001. 2005, 2010.

\subsection{Situación Conyugal de la Población Adulta} Mayor

El estado conyugal predominante es el de casado (a), más de un tercio de la población se encontraba legalmente unido a su pareja, en segundo lugar se ubican los viudos y viudas. Al desagregar los datos por sexo son más las mujeres viudas, por ejemplo de cada cuatro adultos mayores viudos, 3 son mujeres y uno es hombre, esto está en relación directa con el hecho de que mueren más rápidamente los hombres que las mujeres. Un tercer lugar lo ocupa el estado conyugal unión libre, siendo los hombres los que ocupan los porcentajes más altos en esta variable, probablemente algunos de ellos sean viudos que se han unido a nuevas parejas (Gráfico No.7).

Gráfico No. 7. Honduras: Población Mayor de 60 Años, Según Estado Conyugal (En Porcentaje)

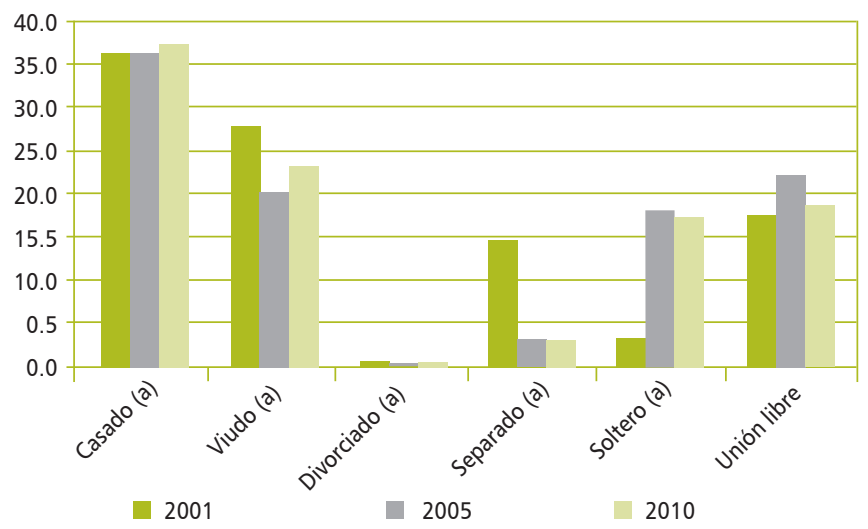

Fuente: Elaborado con datos del INE, EPHPM, 2001. 2005, 2010.

\subsection{Jefatura de Hogar}

Más de dos tercios de la población adulta mayor en Honduras declaró ser jefe de hogar, al desagregar los datos según sexo, son más los hombres adultos mayores que tienen esta condición. De igual manera se observa que generalmente estos hogares sólo están conformados por el jefe de hogar, el cónyuge y en algunos casos viven con otras personas parientes o no parientes.

En el 2001, 64.7\% de los adultos mayores declaró ser jefe de hogar, ya para los años 2005 y 2010 se refleja una leve disminución, 63.8 y $63.7 \%$ respectivamente (Gráfico No.8).

\section{4 Índice de Envejecimiento}

El índice de envejecimiento ${ }^{3}$ de la población permite apreciar la relación numérica que hay entre niños

3. Definido como el cociente entre la población de 60 años y más y aquella menor de 15 años expresada en porcentaje. 
Gráfico No. 8. Honduras: Población Mayor de 60 Años, Según Relación de Parentesco (En Porcentaje)

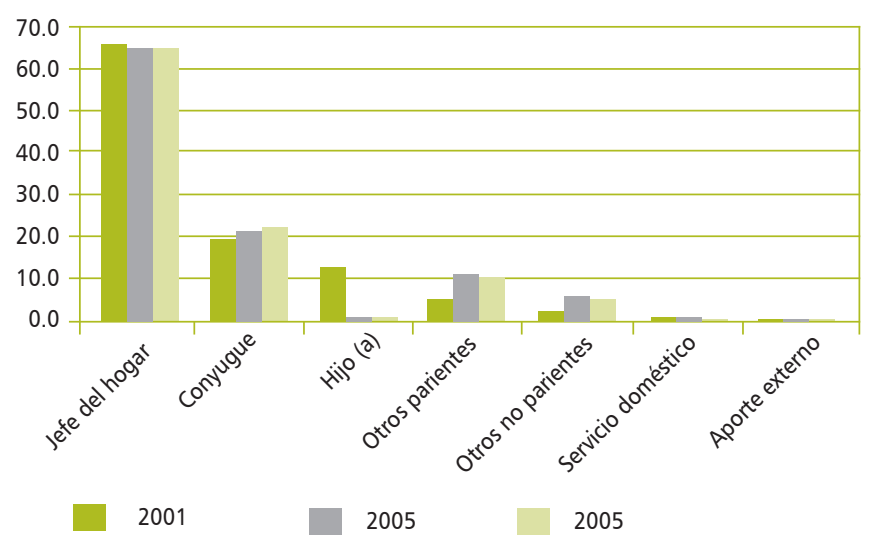

Fuente: Elaborado con datos del INE, EPHPM, 2001. 2005, 2010.

y adultos mayores. En Honduras para el 2001 había 16 adultos por cada 100 niños y niñas menores de 15 años; para el 2005 se incrementó $2.3 \%$ puntos porcentuales y para el 2010, 6.7\% puntos porcentuales (Cuadro No. 1 y Gráfico No.9).

\section{Cuadro No. 1. Honduras: Índice de Envejecimiento}

\begin{tabular}{|c|c|c|c|c|}
\hline Años & $\begin{array}{l}\text { Población < } \\
\text { de } 15 \text { años }\end{array}$ & $\begin{array}{c}\text { Población } \\
>=\text { de } 60 \text { años }\end{array}$ & $\begin{array}{l}\text { Índice de } \\
\text { Envejecimiento }\end{array}$ & Diferencia \\
\hline 2001 & 2621,440 & 426,463 & 16.27 & \\
\hline 2005 & 2843,753 & 529,323 & 18.61 & 2.35 \\
\hline 2010 & 2808,434 & 710,420 & 25.30 & 6.68 \\
\hline
\end{tabular}

Fuente: Elaborado con datos del INE, EPHPM, 2001, 2005, 2010

Gráfico No. 9. Honduras: Índice de Envejecimiento, 2001, 2005 y 2010

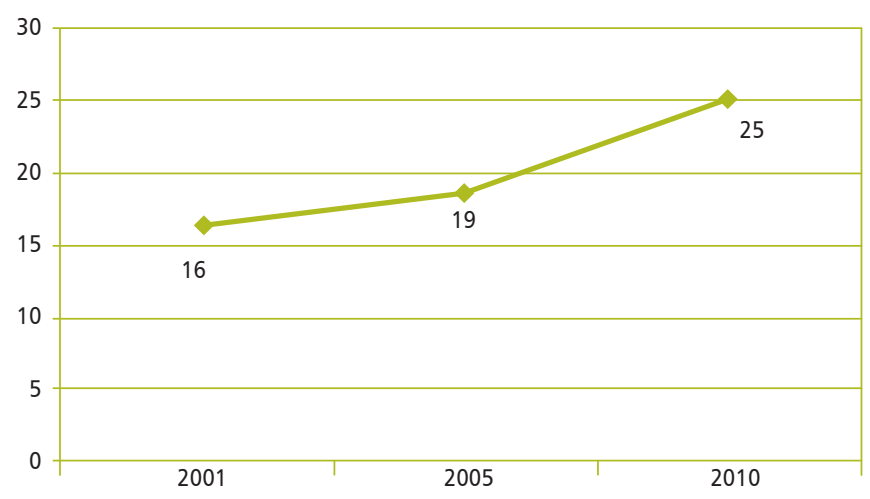

Fuente: Elaborado con datos del INE, EPHPM, 2001, 2005, 2010.
Este índice se incrementará paulatinamente en el presente siglo, y según proyecciones se espera que el número de adultos mayores para el 2050 sea de 68 por cada 100 niños y niñas.

\subsection{Características Educativas de la Población Adul-} ta Mayor

Las características educativas de la población reflejan en alguna medida las condiciones de bienestar, este apartado presenta las características educativas de la población adulta mayor; se analizaron las variables alfabetismo y nivel educativo.

\section{- Alfabetismo}

Se considera alfabeto a todas las personas que al momento de la encuesta contestaron que sabían leer y escribir. Los datos reflejan que un porcentaje muy alto de la población adulta mayor era analfabeta, se observa que en el 2001 este porcentaje corresponde a 49.7\%, para el 2010 presenta una disminución de 6.4 puntos porcentuales, esto probablemente esté relacionado con la implementación de diversos programas de alfabetización que se han desarrollado tanto a nivel nacional como local (Gráfico No.10).

Gráfico No. 10. Honduras: Población Mayor de 60 Años, Según Alfabetismo y Analfabetismo (En Porcentaje)

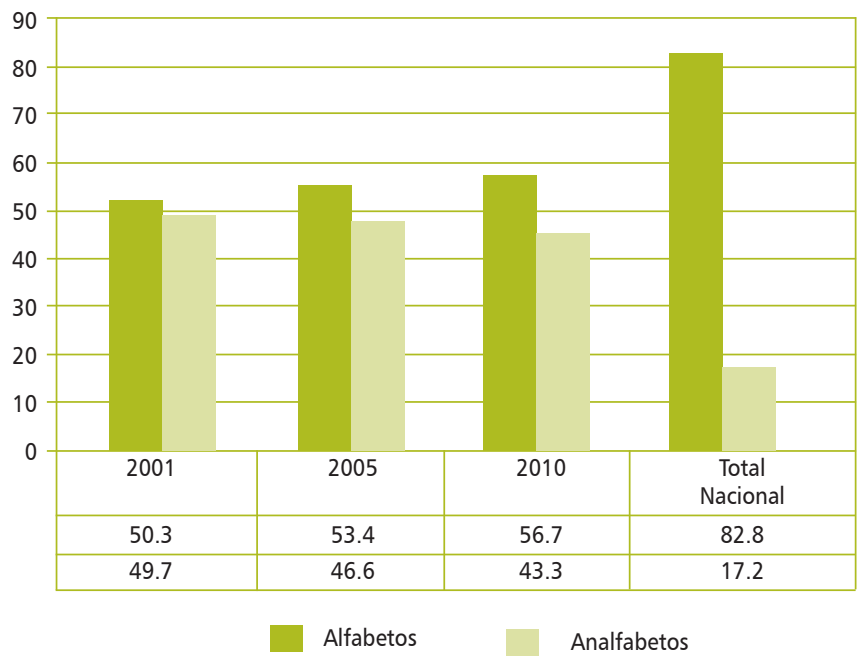

Fuente: Elaborado con datos del INE, EPHPM, 2001. 2005, 2010. 
Al desagregar los datos según sexo, se observa que existe predominio de mujeres analfabetas respecto a los hombres, situación que está directamente relacionada con factores culturales puesto que se creía que las mujeres solamente debían ser formadas en las labores domésticas y ser preparadas para ser buenas esposas y madres.

Los datos reflejan un comportamiento muy similar en los tres periodos objeto de estudio (Gráfico No.11).

\section{Gráfico No. 11. Honduras: Población Mayor de 60 Años,} Según Alfabetismo y Sexo (En Porcentaje)

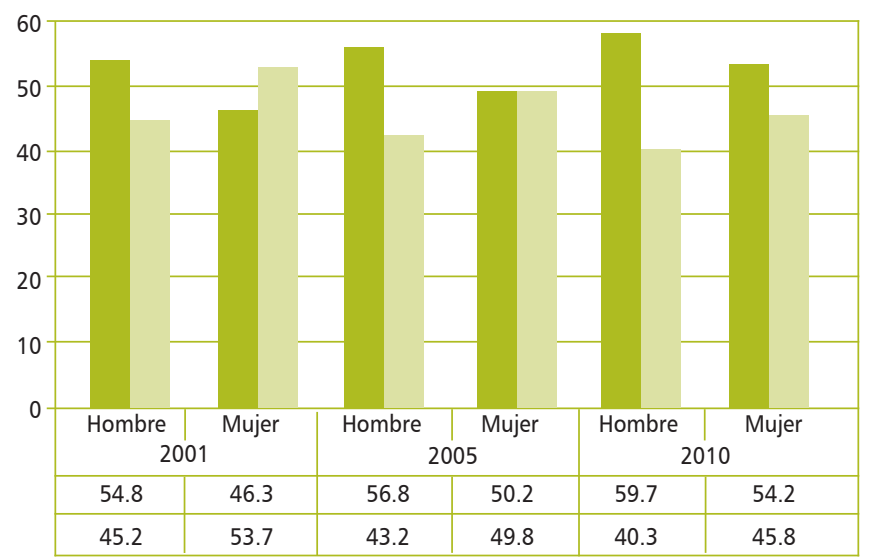

Fuente: Elaborado con datos del INE, EPHPM, 2001. 2005, 2010.

\section{- Nivel Educativo}

Las personas adultas mayores en Honduras se encuentran entre los grupos poblacionales con menor escolaridad, esto motivado por diversos factores entre los que destacan: las escasas oportunidades educativas debido a la baja cobertura del sistema educativo cuando este grupo estaba en edad escolar, las condiciones de pobreza, la incorporación temprana al mercado laboral, la poca importancia que se le daba años atrás a la formación académica, entre otras.

En promedio entre los años 2001 y 2010, 46.6\% de la población adulta mayor no contaba con ningún nivel escolar, lo que representa una diferencia de $24.8 \%$ puntos porcentuales en relación a los datos a nivel nacional. El nivel escolar con mayor cobertura es el de primaria con
$44.3 \%$, seguido del nivel secundario con $6.7 \%$ y apenas 0.4\% realizó estudios de educación superior y en este último caso fueron los hombres los que tuvieron mayor oportunidad (Gráfico No.12).

Cuando los adultos mayores son consultados respecto a las razones que les impiden continuar con sus estudios la mayoría considera estar muy mayor para estudiar, no sentirse motivados para hacerlo o que sus horarios de trabajo se lo impiden.

\section{Gráfico No. 12. Honduras: Población Mayor de 60 Años, Según Nivel Escolar (En Porcentaje)}

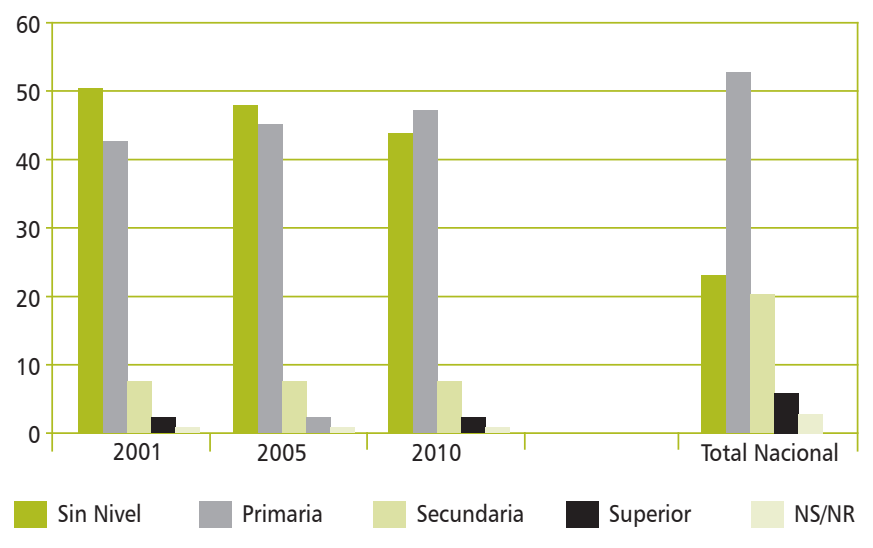

Fuente: Elaborado con datos del INE, EPHPM, 2001. 2005, 2010.

\subsection{Características Económicas}

Al estudiar el tema de envejecimiento no puede quedar de lado las características económicas de este grupo poblacional, puesto que la capacidad de las personas de disponer de bienes en general es fundamental en su calidad de vida en la vejez. La seguridad económica de las personas mayores se define como la capacidad de disponer y usar de forma independiente una cierta cantidad de recursos económicos regulares y en montos suficientes para asegurar su calidad de vida (Guzmán, José M 2003).

Se describe en este apartado: la Población Económicamente Activa (PEA) e Inactiva (PEI), condición de actividad laboral, situación de empleo, rama de actividad e ingresos.

4. PEA Son todas las personas mayores de 10 años que manifiestan tener algún empleo, o bien, no tenerlo pero haber buscado activamente trabajo o buscar por primera vez, está compuesta por ocupados y desocupados.

PEI: Es el conjunto de personas de 10 años y más no clasificadas como ocupadas durante el periodo de referencia 
- Condición de Actividad Económica (PEA y PEI $\left.{ }^{4}\right)$ y Categoría Ocupacional

Los porcentajes más altos se registran entre la PEI, más del 50\% de la población adulta mayor se encontraba inactiva, lo que no implica que goce de seguridad social o que esté completamente desocupada ya que una buena parte de ella se encuentra ocupada en los servicios domésticos familiares, sobre todo en el caso de las mujeres.

Puede observarse que una proporción significativa de los adultos mayores en Honduras es económicamente activa más del $40 \%$, siendo los hombres los que ocupan los mayores porcentajes con $68 \%$, en el caso de las mujeres en promedio sólo el 25\% estaban activas (Gráfico No.13).

La mayor parte de los adultos mayores trabaja por cuenta propia generalmente empleados en el sector informal por lo tanto están excluidos de los beneficios de la seguridad social (Gráfico No.14). Ante la ausencia de oportunidades para el adulto mayor en el mercado laboral, el trabajo por cuenta propia se convierte en una opción que permite cubrir sus necesidades básicas.

La tasa de participación en el caso de la población adulta mayor entre los años 2001 y 2010 , en promedio fue de $46.1 \%$, reflejando que casi la mitad de la población es activa, esto motivado en cierta medida por la carencia de

Gráfico No. 13. Honduras: Población Mayor de 60 Años Según Condición de Actividad y Sexo (En Porcentaje)

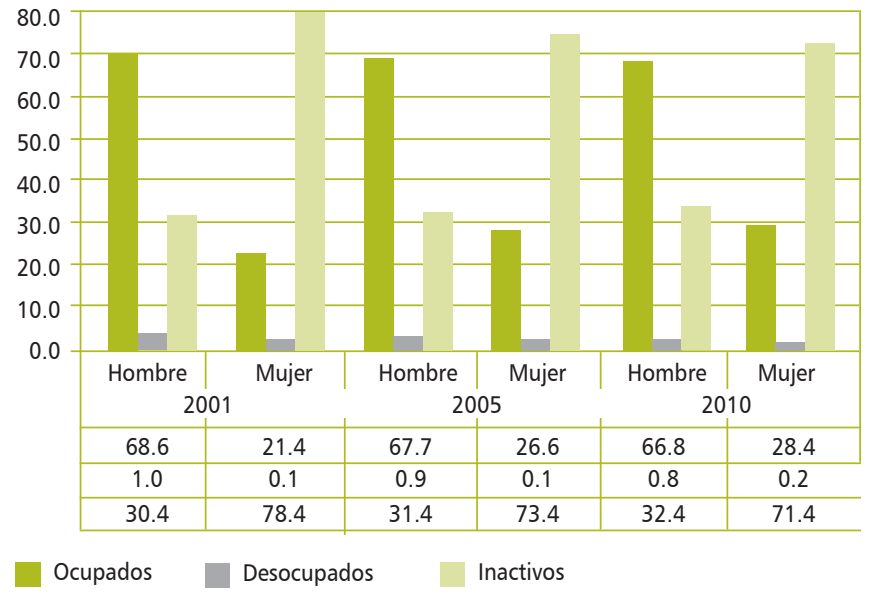

Fuente: Elaborado con datos del INE, EPHPM, 2001, 2005, 2010.
Gráfico No. 14. Honduras: Población Mayor de 60 Años Según Categoría Ocupacional (En Porcentaje)

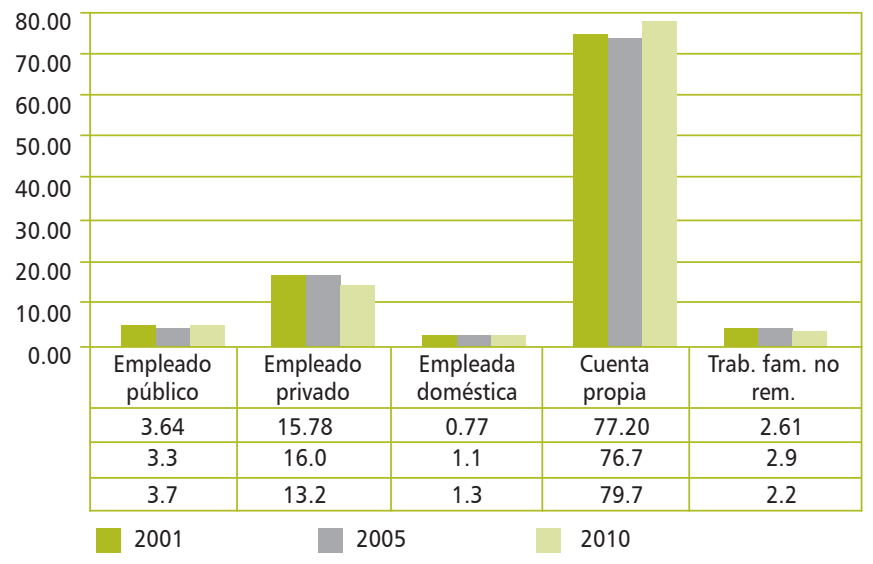

Fuente: Elaborado con datos del INE, EPHPM, 2001, 2005, 2010.

un sistema de jubilaciones y pensiones que le dé cobertura en esta etapa de su vida o porque aquellos que gozan de una jubilación tienen montos muy bajos que no cubren la totalidad de sus gastos (Gráfico No.15).

Se puede observar en el caso de Honduras que un gran porcentaje de las personas adultas mayores se ve en la necesidad de trabajar para obtener un ingreso que permita costear su sobrevivencia diaria.

- Situación de Empleo de los Ocupados

La situación de empleo entre los adultos mayores en

Gráfico No. 15. Honduras: Tasa de Participación Laboral de la Población Mayor de 60 Años (En Porcentaje)

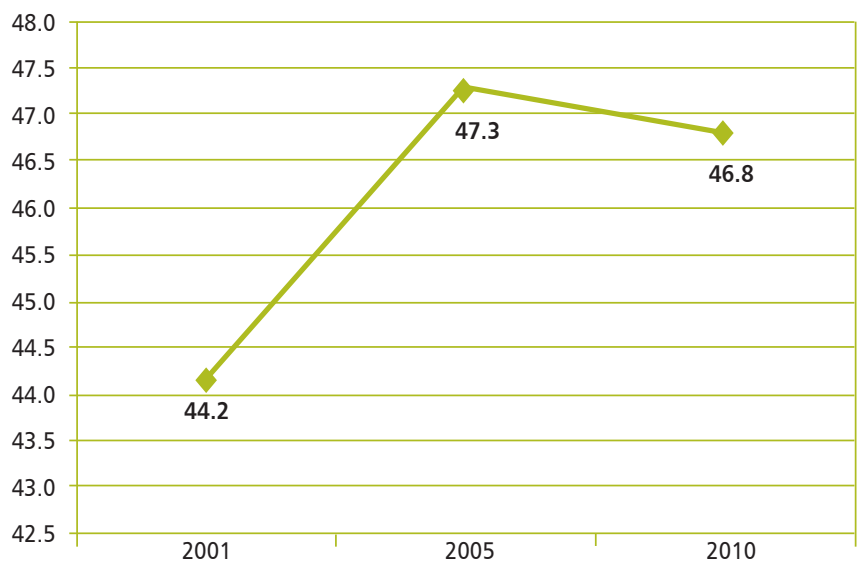

Fuente: Elaborado con datos del INE, EPHPM, 2001. 2005, 2010. 
Honduras refleja que la mayoría de ellos no tiene problemas de empleo esto quizá porque una gran parte trabaja por cuenta propia, sin embargo, es notorio que más de un tercio de la población objeto de estudio está en condición de subempleo invisible ${ }^{5}$, es decir son subempleados, esto aumenta la condición de pobreza en los hogares y su vulnerabilidad social (Gráfico No.16).

Gráfico No. 16. Honduras: Población Mayor de 60 Años, Según Situación de Empleo, (En Porcentaje)

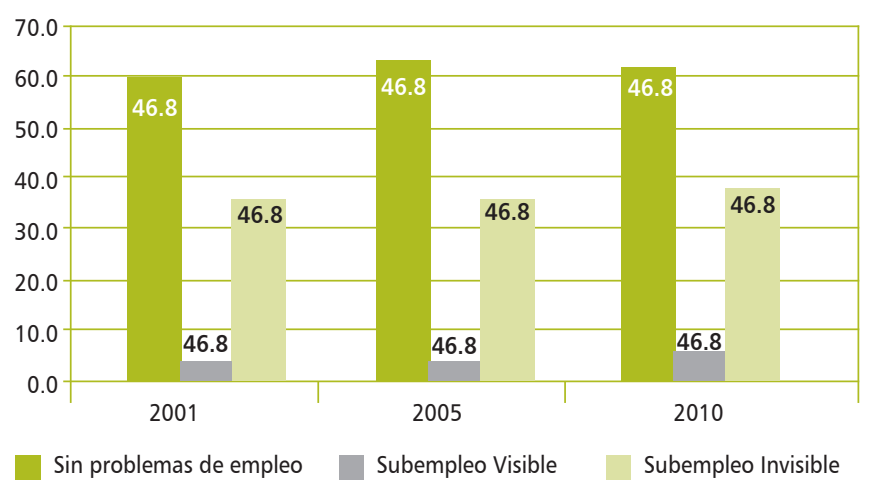

Fuente: Elaborado con datos del INE, EPHPM, 2001. 2005, 2010.
- Rama de Actividad

La población adulta mayor ocupada, mayoritariamente se encuentra desarrollando actividades relacionadas con la agricultura, silvicultura, caza y pesca, esto está directamente relacionado con el hecho que el mayor porcentaje reside en el área rural y desarrolla actividades primarias de la economía, los datos reflejan un incremento de 3.6 puntos porcentuales entre el 2001 y 2010; el segundo lugar lo ocupan las actividades de comercio y un tercer lugar la industria manufacturera (Gráfico No.17).

\section{- Ingresos}

Los ingresos en un hogar determinan en gran medida la calidad de vida de la población. En el caso de los adultos mayores la mayor parte de los ingresos se ubica en ingresos iguales o menores de L. 5,000.00, para el caso en el 2001 el porcentaje correspondía al 90.9\%, en el 2005 al 89.6\% y en el 2010 al 63.15\% en este último caso pudo haber influido la aprobación del salario mínimo realizada en el 2008 por el Ex presidente Manuel Zelaya Rosales sobre todo entre un pequeño grupo de esta población (16.9\%)

\section{Gráfico No. 17. Honduras: Población Mayor de 60 Años, Rama de Actividad (En Porcentaje)}

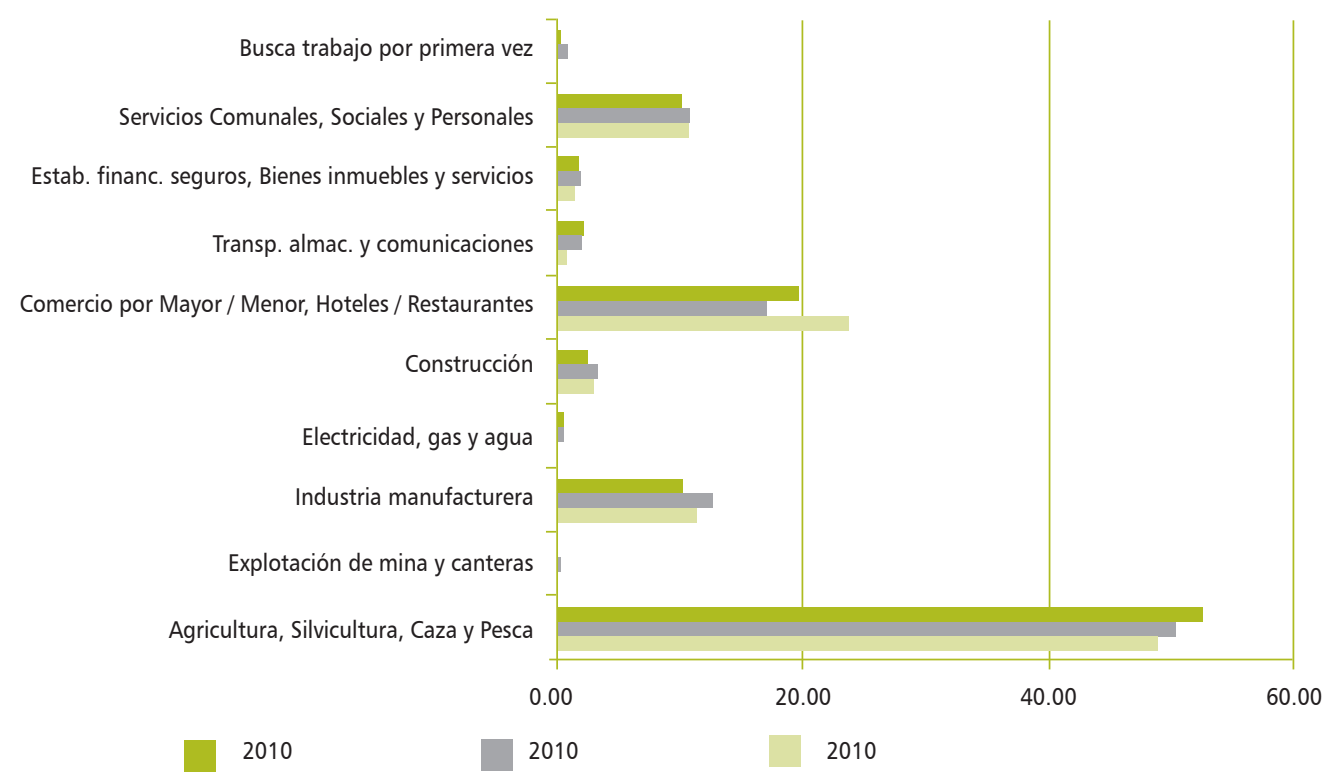

Fuente: Elaborado con datos del INE, EPHPM, 2001. 2005, 2010.

5. Son las personas que habiendo trabajado 36 horas semanales o más, tuvieron ingresos mensuales inferiores al salario mínimo promedio mensual pro rama de actividad económica o área geográfica. 
que trabaja con el sector público y con la empresa privada (Gráfico No.18).

Un detalle que se observa es el hecho de que la mayor parte de los ingresos del adulto mayor proceden del trabajo que realizan por cuenta propia. Entre el 2001 y 2005 en promedio un 38\% de la población adulta mayor tiene ingresos de otras fuentes: bonos $(0.6 \%)$, ayudas particulares (1.55\%), subsidios (2.95\%), jubilaciones (3.35\%), remesas $(9.5 \%)$ y ayudas familiares $(20.5 \%)$.

El contexto anterior refleja que las condiciones de seguridad económica de la población adulta mayor son deficientes lo que aumenta su vulnerabilidad social.

Gráfico No. 18. Honduras: Población Mayor de 60 Años, Según Ingresos por Salario en Ocupación Principal (En Porcentaje)

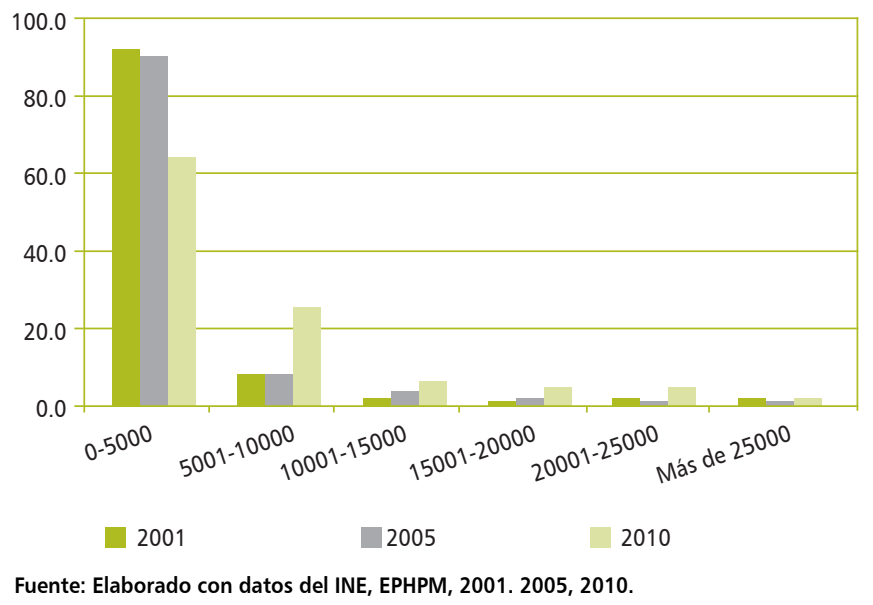

\section{A Manera de Reflexión}

El envejecimiento de la población en Honduras es un fenómeno ligado al proceso de transición demográfica tiene implicaciones sociales y económicas para el país, también representa una oportunidad y un reto para la sociedad. Una oportunidad por cuanto nuestros adultos mayores siguen jugando un papel activo en la economía y en los procesos de socialización comunitarios, de igual manera es un reto ya que sus demandas son diferentes al resto de la población por lo que es necesario que el Estado y los gobiernos locales tomen previsiones ante el crecimiento de este grupo poblacional.
Las características sociodemográficas de la población adulta mayor en el país refleja que este grupo se encuentra en condición de vulnerabilidad; para el caso la mayor parte vive en el área rural donde generalmente las fuentes de trabajo son escasas, más de dos tercios son jefes de hogar lo que implica que tienen responsabilidades económicas que atender.

En relación a las características educativas el 53\% era analfabeta, la mayoría tenía como máximo nivel de educación la primaria y apenas 1.9\% logró el nivel superior universitario, siendo en este último caso los hombres los que tuvieron mayor oportunidad. Respecto a las políticas sociales dirigidas a la educación del adulto mayor, estas deben ir más allá de los programas de alfabetización o de aquella dirigida a su autocuidado y conocimiento de su salud, puesto que los adultos mayores tienen derecho a seguir educándose en las diferentes modalidades. Vale la pena recordar lo que Kofi Anann dijo en el Segunda Asamblea Mundial de Envejecimiento (2002) "En África se dice cuando muere un anciano desaparece una biblioteca", la pregunta obligada es ¿Porqué no aprovechar la sabiduría acumulada por estas personas?. Su cúmulo de conocimientos constituye un aporte valioso que no debe ser menospreciado.

Las características económicas de la población adulta mayor reflejan una alta tasa de participación laboral sobre todo en actividades primarias de la economía y en ocupaciones informales por cuenta propia, más de un tercio está en condición de subempleo invisible y sus ingresos se concentran en rangos menores a los L. 5,000.00. Estas características permiten inferir que la incidencia de la pobreza de los adultos mayores en el país es bastante alta y que afecta en mayor proporción a las mujeres adultas mayores. Pero además muestra la escasa cobertura de seguridad social porque lejos de estar gozando de los beneficios de su retiro, se ven en la necesidad de participar de manera activa en el mercado laboral en muchas ocasiones en condiciones precarias.

En Honduras existe una Ley del Adulto Mayor, sin embargo se considera que esta aún tiene vacíos y que en la práctica muchos de sus derechos son violentados. Y 
aunque uno de los logros de las políticas sociales se refleja en el aumento de la longevidad entre la población esto no sólo debe traducirse en vivir más años, sino también en tener calidad de vida.

\section{Bibliografía}

- CONAPO (2000) el envejecimiento de la población mundial. Transición demográfica mundial. México.

- Guzmán, José Miguel (2003) Seguridad económica en la vejez un aproximación inicial, documento presentado en la reunión de expertos en seguridad económica del adulto mayor (Ciudad de Panamá, 9 al 11 de abril) Banco Interamericano de Desarrollo (BID), Organización Internacional del Trabajo (OIT), Comisión Económica para América Latina y el Caribe (CEPAL).

- $\operatorname{INE}(2001,2005,2010)$ Encuestas Permanentes de Hogares de Proposotos Múltiples, Honduras.

- Villa M, y Rivadeneira (2000) El proceso de envejecimiento de la población de América Latina y el Caribe: una expresión de la transición demográfica. CELADE/CEPAL, Santiago de Chile.

- Population Reference Bureau, 2003. 